\title{
Time structure of individual extensive air showers
}

\author{
M. Ambrosio ${ }^{a, *}$, C. Aramo ${ }^{\text {b,c }}$, L. Colesanti ${ }^{\text {a }}$, A.D. Erlykin ${ }^{\text {d }}$ \\ a INFN, Sezione di Napoli, Italy \\ b INFN, Sezione di Catania, Italy \\ c Dipartimento di Fisica, Università di Catania, Italy \\ d P.N. Lebedev Physical Institute, Moscow, Russian Federation
}

Received 22 September 1998; revised 16 February 1999

\begin{abstract}
Extensive air showers detected by the GREX array have been sampled by means of highly segmented $8 \mathrm{~m}^{2}$ bakelite RPC in the GREX/COVER_PLASTEX experiment. Delay distributions of particles with respect to the first arriving particle in the EAS front at PeV energies have been analysed for individual events in the core distance range of 0-100 m. It is shown that both mean arrival time and EAS front thickness in individual showers fluctuate strongly and cannot be a good measure of the distance from the EAS axis in a $0-100 \mathrm{~m}$ core distance interval.

Individual distributions have been compared with integrated inclusive distributions measured in the same experiment. Results indicate that the width of the individual distribution is systematically less than that of the inclusive distribution. It means that the bulk of particles in individual showers arrive as a relatively compact group delayed by different time intervals from the first arriving particle. Such fluctuations of the arrival time for most of the shower particles may be the consequence of large fluctuations in the shower longitudinal development.

Comparison with CORSIKA Monte Carlo simulations confirmed the difference between the mean width of inclusive and individual arrival time distribution. It revealed also the presence in the experiment of the excessive train of delayed particles near the shower core. This train is obviously due to the non-relativistic low energy hadrons most abundant in the shower core region. (c) 1999 Elsevier Science B.V.
\end{abstract}

\section{Introduction}

Extensive air showers (EAS) in the energy range $10^{14}-10^{17} \mathrm{eV}$ are usually detected by means of ground based scintillator arrays or Cerenkov detectors, the energy threshold and energy range depending on the observation level, array dimension and detector spacing. Primary energy is estimated by the energy deposited in the array detectors, while the shower axis inclination is reconstructed measuring delays between signals in the fired detectors.

\footnotetext{
${ }^{*}$ Corresponding author; e-mail: ambrosio@na.infn.it.
}

Accuracy in the determination of shower axis is particularly important for the detection of high energy $\gamma$ rays from discrete cosmic ray sources with ground based arrays. The improvement of the angular resolution depends strongly on the knowledge of the fine time and space structure of the shower disk, especially near the shower core [1-4]. For the same reason the knowledge of front structure is important for the detection of extremely high energy cosmic rays with large area arrays. In this case array detectors are hundred meters spaced, making difficult the correlation of delays between signals in different detectors without 
taking into account the front profile and thickness.

Unfortunately fluctuations of particle arrival time in the shower front are intrinsic to the phenomenon and can smear out the accuracy of detectors in time measurements. So the determination of the time structure of the shower front is of the prime importance for the improvement of the angular resolution of the ordinary EAS arrays.

Measurement of the EAS disk structure was first attempted by Bassi, Clark and Rossi in 1953 [5] and continued up to date (see [6] and references therein). Connection between measurements close and far from the core was attempted by Linsley [7] on the basis of $[8]$ and $[9,10]$. Linsley's parameterization of the front thickness $\sigma$ is commonly used for correction of delays and improvement of array angular resolution. Some authors reported also modulations in time and sub-structures of EAS front [11-14], making the scenario more and more intriguing.

Previous measurements were all based on the study of integrated delay distributions of particles in the shower front or with respect to the light front. No study has been made on arrival time distribution of particles in the shower front for individual events. The importance of such information is obvious: integrated delay distributions cannot reproduce the behaviour of individual showers, smearing the effect of fluctuations and masking the presence of eventual modulations and structures in the shower front. Then the study of the time structure of individual EAS is particularly important in this scenario. It can permit a better determination of EAS disk structure, a study of fluctuations and correlations between front profile, front thickness and core distance, as well as to reveal the presence of structures on the shower front.

Such a measurement has been made possible with the GREX/COVER_PLASTEX (G/C_P) experiment in Haverah Park, where highly segmented resistive plate counters (RPC) [15,16] were installed inside the GREX array [17] on the top of PLASTEX tracking telescopes [18]. RPCs permitted an accurate arrival time measurement of individual particles in the shower front and the study of correlations between the front profile and thickness versus core distance.

In this paper results of this measurements are reported. Time distributions of the individual events were analysed and compared with inclusive integrated distributions obtained previously in the same exper- iment. Then the measured EAS front thickness was compared with measurements of other authors, and a new parameterization of the shower front has been obtained. Accurate CORSIKA Monte Carlo simulations [19] have been developed and compared with the experimental data. This comparison shows the general agreement between simulated and experimental results. By means of this comparison the influence of low energy hadrons near the core has been found. Results indicate also the presence of events in which most particles arrive delayed with respect to the shower front. Such events cannot be reproduced by simulations.

\section{Experimental apparatus}

Present measurements were made by the G/C_P array located at Haverah Park, near Leeds, UK, situated at $50^{\circ} 58^{\prime} \mathrm{N}, 1^{\circ} 38^{\prime} \mathrm{W}$, at an elevation of $220 \mathrm{~m}$ a.s.l. The triple title of experiment originates from the use of three relatively independent parts, with different kind of detectors, built in different times.

\subsection{The GREX array}

The GREX array, managed by the University of Leeds [17], consisted of $36 \times 0.84 \mathrm{~m}^{2}$ scintillation detectors, 14 of them arranged in a hexagonal outer grid with $50 \mathrm{~m}$ baseline separation and 22 in an inner grid with $30 \mathrm{~m}$ separation. The array covered a total area of $3.5 \cdot 10^{4} \mathrm{~m}^{2}$ and had an energy threshold of about $4 \cdot 10^{14} \mathrm{eV}$. The trigger signal was built by a coincidence of at least five scintillators fired with an energy deposit corresponding to more that 1 minimum ionizing particle (m.i.p.). The arrival direction of the primary particle was measured in the traditional way, as the axis normal to the shower front plane derived from the relative time signal onset in the scintillators. The high time resolution of detectors ensured a precision of the shower axis reconstruction better than $1^{\circ}$. The EAS core position was evaluated on the basis of the circular symmetry of the particle density in the shower front. A charge integrating ADC channel measured, for each scintillator, the signal amplitude up to a maximum ( saturation) limit corresponding to 45 m.i.p. The accuracy of the core location was $\sim 6 \mathrm{~m}$ in the inner part of the array and $\sim 9 \mathrm{~m}$ at the pe- 
riphery, i.e beyond $\sim 60 \mathrm{~m}$ from the array center. The primary energy $\mathrm{E}_{0}$ was estimated from $S_{50}$ - the particle density at a distance of $50 \mathrm{~m}$ from the core. The density at this distance had a good correlation with $E_{0}$ and was relatively insensitive to variations of the shower age.

\subsection{The PLASTEX sub-array}

The PLASTEX sub-array, managed by the Palermo IFCAI (Istituto di Fisica Cosmica e Applicazioni all'Informatica - CNR), was made of 4 plastic limited streamer tube (LST) hodoscopes [18], two of them, named $A \& B$, placed side by side at about $4 \mathrm{~m}$ from the geometric center of GREX and the other two, named $\mathrm{C} \& \mathrm{D}$, located at the same distance $(150 \mathrm{~m})$ from the array center [6]. Each hodoscope was a stack of two identical tracking chambers, one above the other, with a thin layer of high $\mathrm{Z}$ absorber between them [6]. Each chamber consisted of three nearly square $\sim 6$ $\mathrm{m}^{2}$ LST planes with $X$ and $Y$ readout strips on each plane. The purpose of this sub-array was to identify electrons, photons and muons in the shower disk.

\subsection{The COVER sub-array}

On the top of each LST hodoscope a $4 \mathrm{~m}^{2}$ layer of single gap bakelite RPC $[15,16]$ was installed with the aim to measure the arrival time of individual particles crossing the PLASTEX hodoscopes. RPCs of $1 \times 2 \mathrm{~m}^{2}$ size and $10^{12} \Omega \mathrm{cm}^{2}$ resistivity were operated with a gas mixture 68\% Argon, 28\% Isobuthane, 4\% Freon, and covered a total area of $16 \mathrm{~m}^{2}\left(8 \mathrm{~m}^{2}\right.$ in the array center, $4+4 \mathrm{~m}^{2}$ in the array periphery). The signal pick-up was obtained by means of $12 \times 12 \mathrm{~cm}^{2}$ copper pads, small enough to avoid the time jitter due to the signal propagation. In order to reduce the number of pick-up readout channels, 4 pads was ORed together by means of a pure resistive $\mathrm{OR}_{4}$. In total $4 \times 64=256$ timing channels were installed, 128 in the array center and $64+64$ in the array periphery, so allowing detection of up to 32 individual particles $/ \mathrm{m}^{2}$ per $16 \mathrm{~m}^{2}$. When operated in connection with tracking LST telescopes, RPCs allow a separate measurement of arrival time of electrons and muons in the shower front.

Calibration runs were made periodically using LST tracking hodoscopes: a coincidence between the upper and lower planes selected atmospheric muons cross- ing the hodoscope. In this way detector characteristics were continuously monitored, in particular for what concerns a single counting rate, plateau, efficiency, cross talk and after pulses. A noise level due to accidental coincidences of $1.5 \cdot 10^{-6} \mathrm{~ns}^{-1}$ per each $4 \mathrm{~m}^{2}$ layer was found. When the signal threshold was set to $120 \mathrm{mV}$ at $7200 \mathrm{~V}$, then a minimum energy of 3 $\mathrm{MeV}$ was required for electrons of shower front to cross the RPC pick up and bakelite wall, and generate a detectable signal. Temperature and pressure effects were found to be negligible $[20,21]$.

\subsection{The RPC readout electronics}

While RPC signals arrive few ns after the shower front crossing the detector, the array trigger arrives with a delay of about $600-800 \mathrm{~ns}$. Then electronics readout boards must work in free running mode. For this purpose a new front-end electronics, named T\&T (Tracking \& Timing), was developed [22,23]. This electronics allows a local signal processing and acquisition independent from array trigger: the input signal in each channel crosses a 16 output passive delay, $2 \mathrm{~ns}$ delay per output; a 16 bit cyclic memory continuously shifting stores the passive delay output image also in the absence of signal, with a $32 \mathrm{~ns}$ frequency. The cycle is stopped by the trigger signal, also stored in a T\&T memory. In this way the arrival time of a signal in each channel with respect to the array trigger can be evaluated simply by counting the number of bits between the signal image and the trigger image with a $2 \mathrm{~ns}$ accuracy ( $1 \mathrm{bit}=2 \mathrm{~ns}$ ). The acquisition program scans, only for fired channels, a $1.5 \mu$ s memory depth before the trigger arrival in the A\&B RPC layers and $2.5 \mu \mathrm{s}$ in the $\mathrm{C}$ and $\mathrm{D}$ layers. In these time window the history of the shower front is recorded, with a negligible background due to accidental coincidences of $1.5 \cdot 10^{-6} \mathrm{~ns}^{-1}$ per layer [21]. Taking into account the intrinsic detector response, the total time resolution of RPC + electronics is about 2 ns [20].

\section{Analysis of experimental data and simulations}

On the analogy with measurements of the EAS front profile and thickness measured previously by the G/C_P experiment [6], data analysis has been restricted for only events detected by the two RPC de- 
tector layers $\mathrm{A} \& \mathrm{~B}$ in the array center, where up to 128 independent arrival times in a single event can be measured. Times are corrected taking into account the shower axis inclination with respect to the RPC layers and referred to the first arriving signal. Then, after applying the same cuts and analysis criteria, results of the present work on arrival time distributions in individual events can be directly compared with those on integrated inclusive arrival time distributions presented in [6].

\subsection{Event selection criteria}

The arrival time of particles in each shower was measured with respect to the arrival time of the array trigger and then referred to the earliest particle arrival time in the detector. So the study of individual arrival time distributions of particles with respect to the first arrival was possible when the number of fired pads was $m \geq 2$. However, a lower threshold of 13 fired pads ( $\geq 2$ particles $/ \mathrm{m}^{2}$ ) has been applied to cut events with poor individual statistics. Also an upper limit of 60 fired pads $\left(\leq 7\right.$ particles $\left./ \mathrm{m}^{2}\right)$ has been applied to reduce the probability of more than 1 particle hitting a single pad, and avoid saturation problems [21]. This requirement leads to a non-uniform sampling in shower size, enhancing the fraction of more energetic showers at larger core distances. Within this limit the time delay of each fired pad with respect to the earliest time measured can be assumed to be the time delay of each particle with respect to the first incoming particle in the detector.

Since in the present work the correlation between arrival time distributions and the core distance is studied, an accurate measurement of the core location is mandatory for the analysis. The reliability of the GREX estimate worsens as the real core position approaches the array periphery. In order to reduce the core location ambiguities, the event sample has been cleaned up cutting events with low density in scintillators (maximum density $<5$ m.i.p.), events in which a peripheral detector is in saturation ( $>42$ m.i.p.) or shows the higher density and events in which more than 3 scintillators are in saturation. In this way we eliminate the inclusion of the rather far shower cores, the probability of which we estimate to be less than $10^{-2}$. In addition analysis has been restricted to only events whose core position was inside the inner part of the array, i.e. no more than $100 \mathrm{~m}$ from the RPC layers $\mathrm{A} \& \mathrm{~B}$, taking into account the inclination of the shower axis. In this conditions the distribution of core locations within the selected area is uniform and the core location accuracy of GREX array in selected events is assumed to be $6 \mathrm{~m}$.

On the basis of previous results reported in [6] no cuts have been applied on the shower size or on incidence zenith angles and the average time window for arrival times has been set to $600 \mathrm{~ns}$ to avoid systematic electronics effects. A total of $\sim 190000$ events survive cuts.

\subsection{Data analysis criteria}

Under selected conditions each event $k$ is characterized by

- particle multiplicity $m(13 \leq m \leq 60)$;

- $m$ time measurements $t_{i}^{k}, i=1, m$;

- $N=m-1$ delays $\tau_{i}^{k}=t_{i}^{k}-t_{1}^{k}$ with respect to the first (earliest) particle arriving in the RPC detectors;

- $R=$ distance of core position from the RPC layers $\mathrm{A} \& \mathrm{~B}$;

- axis inclination $\theta$ and $\phi$;

- estimated primary energy $E_{0}$.

For each event $k$ a $\tau_{i}^{k}$ delay distribution with respect to the first arriving particle is obtained. The average arrival delay $\left\langle\tau_{i}^{k}\right\rangle$ and disk thickness $\sigma^{k}$ of individual showers have been estimated as

$$
\begin{aligned}
\left\langle\tau_{i}^{k}\right\rangle & =\sum_{i=1}^{N} \tau_{i}^{k} / N, \\
\sigma^{k} & =\sqrt{\sum_{i=1}^{N}\left(\tau_{i}^{k}-\left\langle\tau_{i}^{k}\right\rangle\right)^{2} /(N-1)} .
\end{aligned}
$$

In this way the delay of the first arriving particle is not accounted for in the distribution.

It must be emphasized that this is the distribution of the delay time with respect to the first particle "locally detected", which is not the same as the arrival time distribution of the "light front", i.e. the plane crossing the detector perpendicularly to the shower axis. Nevertheless the performed simulation (Section 3.3.1) demonstrated that in the selected particle density range the plane light front coincides with the first arriving particle within experimental errors at any core distance 


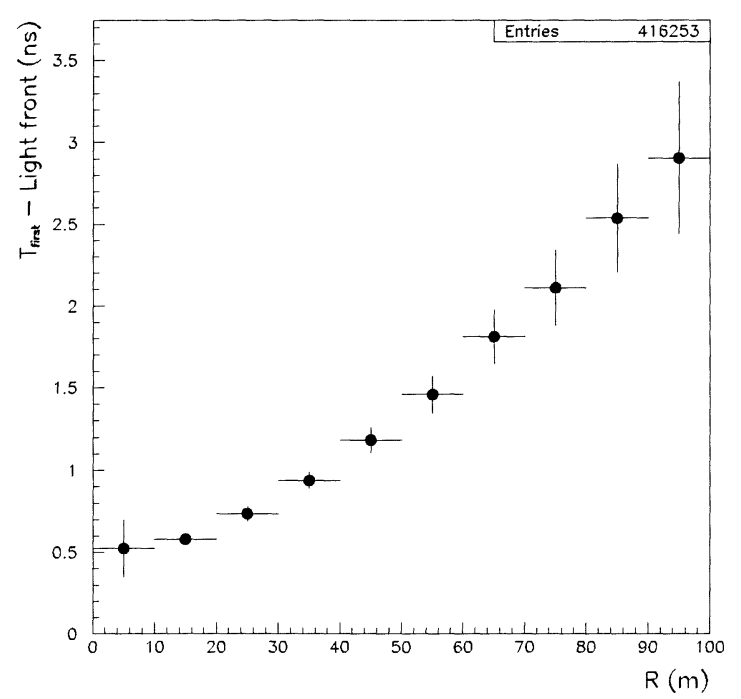

Fig. 1. Delay (ns) of first particle detected with respect to the light front vs core distance $R(\mathrm{~m})$.

(Fig. 1). This result can be understood by the primitive estimation of the delay as $\tau_{i}=R^{2} / 2 h c$, where $h$ is the muon production height. Because the first arriving particle is mostly relativistic muon originated $h \sim 5 \mathrm{~km}$ above the sea level, a delay $\tau \sim 3 \mathrm{~ns}$ must be expected at a core distance $R=100 \mathrm{~m}$. Therefore, analysed delay distributions can be considered as particle arrival time distributions.

In order to estimate experimentally the fluctuation of this "zero" time, the arrival time of the first particle detected in stack A has been compared with the corresponding time of the first particle detected in stack B. It has been found that the time difference depends on the pad multiplicity and the core distance, being in any case of the same order as the electronics time resolution ( $2 \mathrm{~ns}$ ) at any core distance. This means that in the chosen range of multiplicity and core distances the experimental "zero" time corresponds to the shower front arrival time within the accuracy of timing readout electronics, and delay distributions can be treated as arrival time distributions of particles in the shower front.

\subsection{Simulations}

Accurate simulations have been performed using the CORSIKA 4.50 Monte Carlo code. Showers were generated in the energy range of GREX array 0.25-
$10 \mathrm{PeV}$ for five primary groups $(\mathrm{P}, \mathrm{He}, \mathrm{M}, \mathrm{H}, \mathrm{VH})$ with $0^{\circ}$ and $30^{\circ}$ zenith angle. A total of 870 showers have been generated, 174 for each mass group. VENUS + EGS code have been used for hadronic and electromagnetic interactions, respectively. Particle energy thresholds have been taken as $50 \mathrm{MeV}$ for muons and hadrons and $3 \mathrm{MeV}$ for electrons, positrons and photons. These thresholds are minimum energies recommended for the use by CORSIKA authors. The effect of a threshold in the simulations higher than the experimental threshold for muons and hadrons can origin misunderstandings or suggest wrong conclusions. Nevertheless, as it will be seen from the comparison of simulations with the experiment, performed simulations are adequate for the vast majority of events. Minor exceptions will be examined later.

Simulated showers have been combined assuming an uniform primary mass composition and a power energy spectrum with the differential slope index -2.6 in the $0.25-2.5 \mathrm{PeV}$ energy range and -3.05 at $2.5-$ $10 \mathrm{PeV}$. Inclined showers have been added to vertical showers with a weight of $\cos 30^{\circ}$.

\subsubsection{Simulated shower analysis}

Simulated showers have been analysed according to the experimental analysis criteria. It has been supposed that each shower impact at the center of a continuous carpet of $204 \times 202=4 \times 10^{4} \mathrm{~m}^{2}$ area, made of $51 \times$ $101=5151$ detectors of $4 \times 2=8 \mathrm{~m}^{2}$ each. Arrival time of individual particles in the shower front are stored in a matrix element corresponding to hitted detectors. At the end of this process, each detector simulates the real apparatus at a different core distance. Then cuts are applied on multiplicity and core distance as in the experimental data analysis. For the surviving detectors delay distributions are obtained according to (1) and (2). The process is repeated for three conditions:

(1) no inaccuracy on arrival time measurement and on core position;

(2) no inaccuracy on arrival time measurement and $6 \mathrm{~m}$ accuracy on core position;

(3) 2 ns accuracy on arrival time measurement and $6 \mathrm{~m}$ accuracy on core position.

The first condition corresponds to the "ideal case" of an apparatus with no experimental errors (precise measurements of $t_{i}$ and $R$ ), and allows the comparison of experimental results with theory. The second 


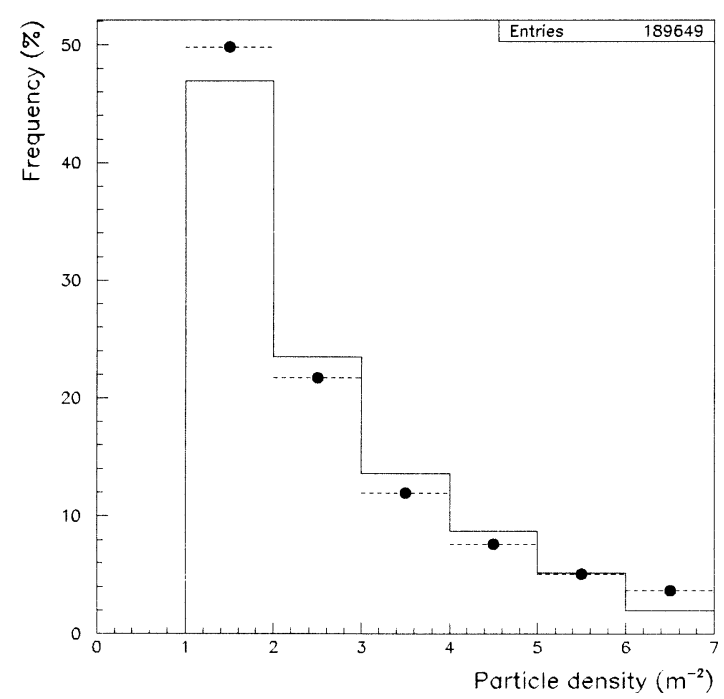

Fig. 2. Comparison between experimental particle density (full line) and simulated particle (broken line).

condition allows the study of effects of the core position accuracy on arrival time distributions. The last condition corresponds to the experimental apparatus: simulated distributions are treated exactly as the experimental ones. 416253 simulated events covering the G/C_P energy range survive analysis cuts.

A separate analysis of simulated showers has been performed also grouping events for primary energy, primary mass, axis inclination and particle multiplicity bins. In addition the contribution of electrons, muons and charged hadrons to delay distributions has been investigated.

Comparison with experimental particle density distribution indicates that the simulation and its analysis reproduce experimental conditions quite well, taking into account that pick-up pads are $24 \times 24 \mathrm{~cm}^{2}$ sized and then the probability to have two particles in the same pad is not negligible. Fig. 2 shows how simulated particle density distribution fits the experimental one.

\section{Results}

The time structure of the shower disk for individual events has been studied separately in the core distance intervals $0-10 \mathrm{~m}, 10-20 \mathrm{~m}, 20-30 \mathrm{~m}, \ldots$ etc., up to $100 \mathrm{~m}$. Then analysis has been made of correlations with the particle multiplicity and primary energy. The influence of experimental accuracy has been estimated from simulations, as well as contribution of different kind of particles in the shower disk.

\subsection{Arrival time distributions}

Fig. 3a shows the average arrival time $\left\langle\tau_{i}^{k}\right\rangle$ distributions of individual particles in individual events from experimental and simulated showers for three different core distance intervals: $0-10 \mathrm{~m}, 40-50 \mathrm{~m}$ and 90 $100 \mathrm{~m}$. The presence of events with high $\left\langle\tau_{i}^{k}\right\rangle$, more than $60 \mathrm{~ns}$, is evident both in experimental and in simulated data. Consequently, it is not surprising to see events with a large disk thickness $\sigma^{k}$, more than $60 \mathrm{ns,}$ shown in Fig. 3b. Distributions appear to be similar to those obtained for integrated arrival time measurements, reported in [6], in which the bulk of distribution is followed by a long "train" of delayed particles.

It can be seen that simulations do not reproduce exactly experimental distributions, mainly near the shower core. Analysis of simulated showers demonstrates also that events with high $\left\langle\tau_{i}^{k}\right\rangle$ and events with high $\sigma^{k}$ are mainly due to the presence of low energy hadrons in the shower front.

The presence of a long "train" of delayed particles in the shower front is more evident from Figs. 4a and $4 \mathrm{~b}$, where $\left\langle\tau_{i}^{k}\right\rangle$ and $\sigma^{k}$ are plotted versus the core distance $R$ for experimental (upper plots) and simulated events (lower plots). Most of the events present a narrow arrival time distribution, but it is clearly seen that some events appear with high $\left\langle\tau_{i}^{k}\right\rangle$ and/or large $\sigma^{k}$. The weak correlation between $\left\langle\tau_{i}^{k}\right\rangle, \sigma^{k}$ and $R$ is confirmed by simulations, as shown in lower plots of Fig. 4. Fluctuations appear to be lower than in the experimental data, mainly for $\sigma^{k}$, and not due to the limited experimental accuracy of arrival time measurements or to an inaccuracy of the shower core estimation: corresponding plots in "ideal" conditions are essentially the same.

The wide spread of individual $\left\langle\tau_{i}^{k}\right\rangle$ and $\sigma^{k}$ sets the limits on the possibility to use them as a measure of the shower core distance. The idea of such measurements was based on the rise of the mean $\Sigma=\left\langle\sigma^{k}\right\rangle$ with the increasing core distance [7]. At core distances as large as $R>50 \mathrm{~m}$ this rise is strong enough to enable the measurement of $R$ if fluctuations were not high. However, as it is seen in Fig. 7 actual fluctuations are 
a)
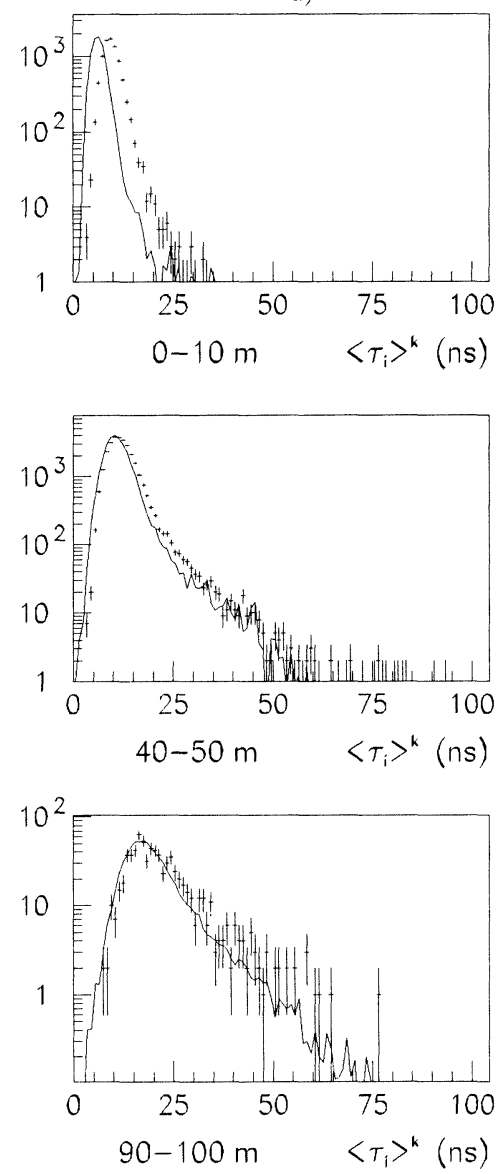

b)
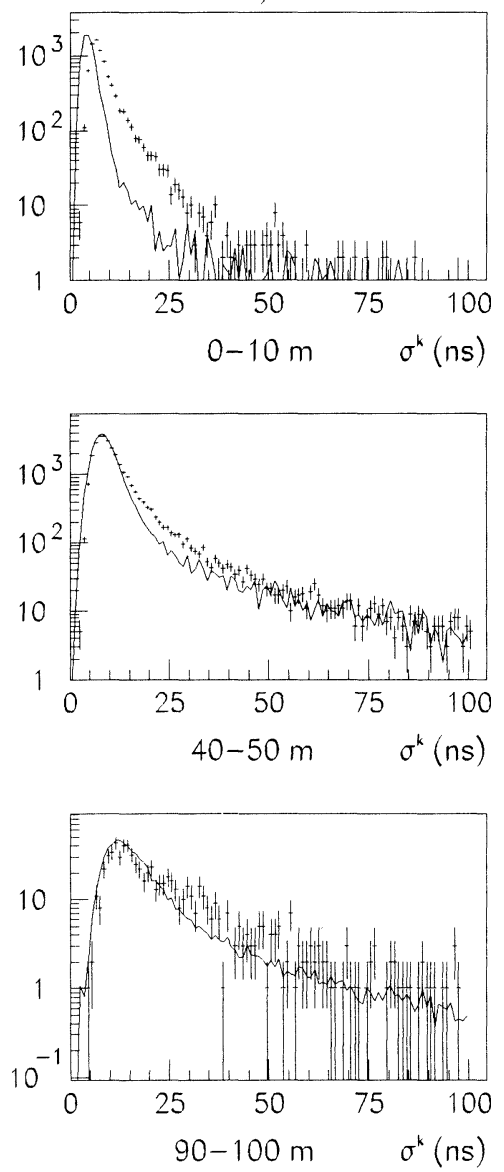

Fig. 3. (a) Average arrival time $\left\langle\tau_{i}^{k}\right\rangle$ and (b) average disk thickness $\sigma^{k}$ of individual events at core distances $R=0-10 \mathrm{~m}, R=40-50 \mathrm{~m}$ and $R=90-100 \mathrm{~m}$ for experimental (points with errors) and simulated (full histogram) events.

large to disprove this idea at least for distances $R<$ $100 \mathrm{~m}$.

\subsection{Shower front characteristics}

The shower disk at the observation level appears as a cloud of particles arriving with an arrival time distribution that can be described by means of a $\Gamma$ probability distribution function ( $\Gamma$-p.d.f.) [6]. It might be expected that there is a strong correlation between the particle average arrival time $\left\langle\tau_{i}^{k}\right\rangle$ and the front thickness $\sigma^{k}$ : as $\left\langle\tau_{i}^{k}\right\rangle$ increases $\sigma^{k}$ should increase as well.

In Figs. 5a,b the scatter plot $\sigma^{k}$ vs $\left\langle\tau_{i}^{k}\right\rangle$ derived from experimental distributions is presented both as the scatter plot (a) and as the lego plot (b). The full line in the scatter plot indicates the $\sigma^{k}$ vs $\left\langle\tau_{i}^{k}\right\rangle$ relation derived from inclusive distributions [6]. The correlation between $\sigma^{k}$ and $\left\langle\tau_{i}^{k}\right\rangle$ is evident, but it must be noted that the bulk of events have $\sigma^{k}$ below the mean $\sigma$ derived from inclusive distributions. In addition there are events with large $\left\langle\tau_{i}^{k}\right\rangle$ but relatively small $\sigma^{k}$. No events have the opposite ratio: small $\left\langle\tau_{i}^{k}\right\rangle$ and large $\sigma^{k}$. It means that there are showers in which most of particles arrive by the compact group late after the first particle. This observation is more evident for the lego plot.

In [24] evidence was reported that among all the showers detected in G/C_P experiment about 2-3\% show the presence of so-called "delayed showers", i.e. events in which correlated particles in all the three 
a)

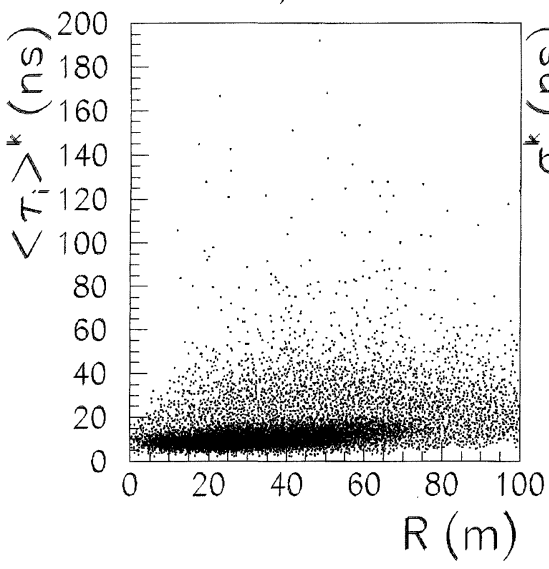

b)
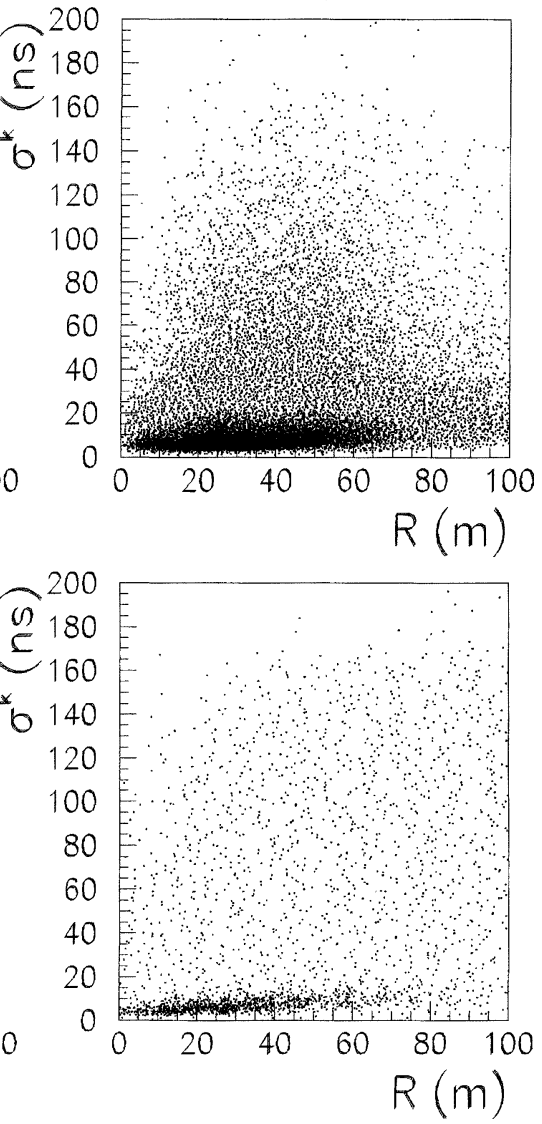

Fig. 4. (a) Average arrival time $\left\langle\tau_{i}^{k}\right\rangle$ and (b) average disk thickness $\sigma^{k}$ vs core distance $R$ for experimental (upper plots) and simulated (lower plots) events.

RPC layers (A\&B, C, D) appear delayed more than the time $t_{i}=\langle\tau\rangle_{R}+3 \sigma_{R}$, where $\langle\tau\rangle_{R}$ and $\sigma_{R}$ are the mean arrival time and the standard deviation of inclusive delay distributions at the core distance $R$. The $\sigma^{k}$ vs $\left\langle\tau_{i}^{k}\right\rangle$ region in the scatter plot of Fig. 5a corresponding to delayed showers is that restricted by the two broken lines and broken-dotted line; it is impressive to find in this area the presence of events with large $\left\langle\tau_{i}^{k}\right\rangle$ and relatively small $\sigma^{k}$.

Much more impressive is the complete absence of such events in simulated showers. Figs. 5c,d show the same scatter and lego plots obtained from simulations. Simulated showers, analysed in the described way (Section 3.3.1), correspond to 870 showers and 416253 quasi-experimental detector events. If $\left\langle\tau_{i}^{k}\right\rangle$ and $\sigma^{k}$ are independent for different detectors in the same shower, then almost 8000 events are expected in the delayed shower area, 210 among those must appear in the restricted area. The complete absence of such events in simulations means that the origin of these events needs additional theoretical study. The most likely explanation is that $\left\langle\tau_{i}^{k}\right\rangle$ and $\sigma^{k}$ correlate in the shower so that observed delayed events are due to the fluctuations in the longitudinal development which influences both $\left\langle\tau_{i}^{k}\right\rangle$ and $\sigma^{k}$ at all distances.

\subsection{Space-time correlation}

Space-time correlation in the shower disk can be studied by measuring the average arrival time and average front thickness as the function of the core distance $R$. In Figs. 6a,b the dependence on $R$ of $T$, mean 

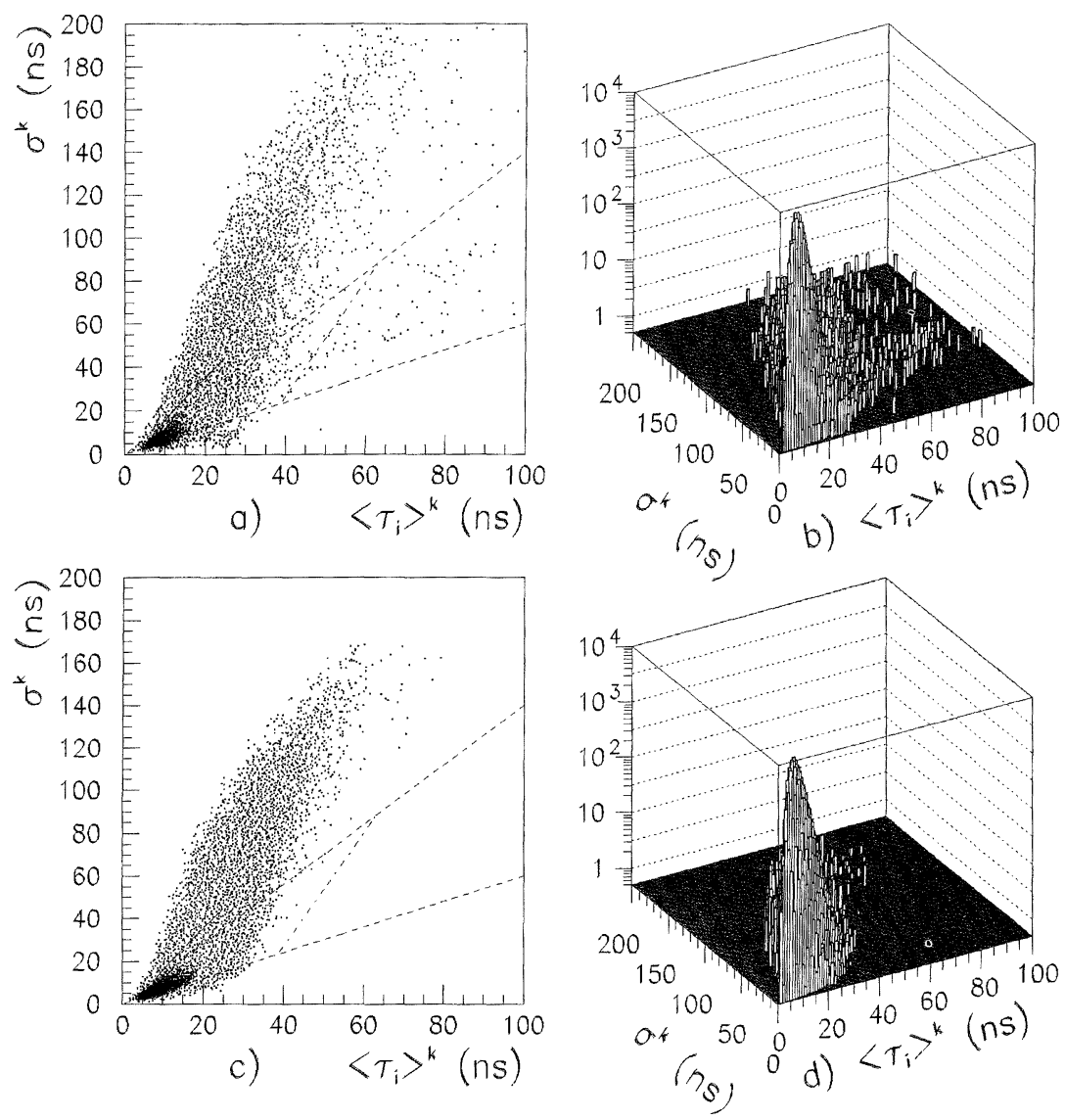

Fig. 5. Average disk thickness $\sigma^{k}$ vs average arrival time $\left\langle\tau_{i}^{k}\right\rangle$ for (a) experimental and (c) simulated events. Right plots (b) and (d) are the same presented as lego plots.

value of $\left\langle\tau_{i}^{k}\right\rangle$ and $\Sigma$, mean value of $\sigma^{k}$, is reported and compared with that obtained from simulations.

Lines drawn through points of the graph are the best fits obtained using, in analogy with fits of integrated distributions in [6], the following expression,

$T, \Sigma=f+g\left(\frac{R}{R_{M}}\right)^{h}$,

with the Molière radius $R_{M}=79 \mathrm{~m}$. The values of the best fit parameters for the experiment and simulations are shown in Table 1.

It can be seen that the mean $T$ of arrival time distributions in individual events increases from 9 to $22 \mathrm{~ns}$ in the selected core distance range of 0-100 m, while the average front thickness $\Sigma$ ranges from 8 to $25 \mathrm{~ns}$, both quite flat up to $30 \mathrm{~m}$. The comparison with simulations reveals a significant difference at the small
Table 1

Best fit parameters for experimental and simulated values of $T$ and $\Sigma$ in expression (3)

\begin{tabular}{llrl}
\hline Parameter & \multicolumn{1}{l}{$f$} & \multicolumn{1}{l}{$l$} \\
\hline$T_{\exp }$ & $8.66 \pm 0.02$ & $9.19 \pm 0.07$ & $2.02 \pm 0.02$ \\
$\Sigma_{\exp }$ & $7.80 \pm 0.04$ & $11.28 \pm 0.14$ & $2.03 \pm 0.04$ \\
$T_{\text {sim }}$ & $6.19 \pm 0.02$ & $11.15 \pm 0.03$ & $1.32 \pm 0.01$ \\
$\Sigma_{\text {sim }}$ & $5.02 \pm 0.06$ & $14.46 \pm 0.08$ & $1.41 \pm 0.02$ \\
\hline
\end{tabular}

core distance, while in the middle results are consistent. The discrepancy can be due to the presence in the experimental data of particles with an energy below the threshold applied in the simulations, in particular the low energy hadrons. In order to investigate better the space-time correlation and to find the cause of discrepancy, both experimental and simulated data 

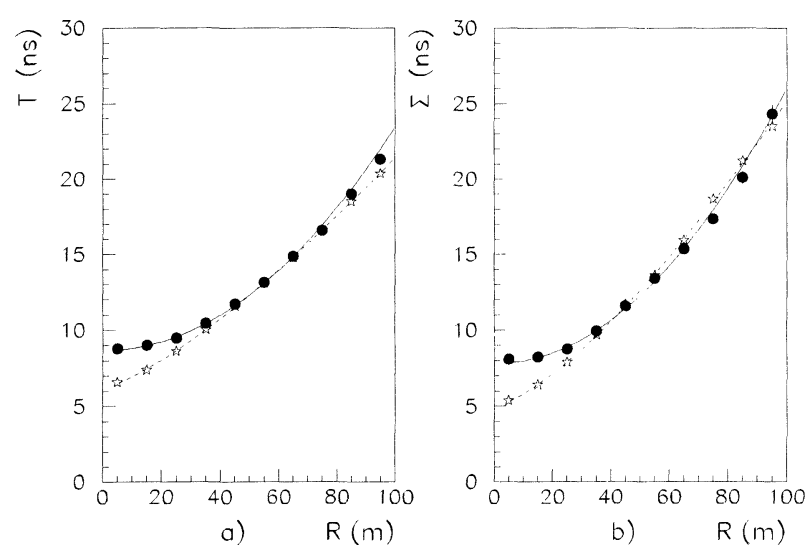

Fig. 6. (a) Mean arrival time $T$ and (b) mean disk thickness $\Sigma$ derived by averaging individual $\left\langle\tau_{i}^{k}\right\rangle$ and $\sigma^{k}$ for experimental data $(\bullet)$ and simulations $(\star)$ as a function of the EAS core distance $R$. The lines drown through the points are the best fit obtained using function (3).

have been analysed as the function of the particle density and the primary energy. No significant difference has been found both for mean arrival time and mean disk thickness for the different particle density, different primary energy and shower axis inclination within the studied core distance range and for the experimental time resolution of the electronics. Also no difference has been found for the different primary nature in the simulated showers. With the help of simulations also the influence of experimental inaccuracy on shower core position and electronics time resolution has been investigated. Figs. 7a,b show how the mean arrival time $T$ and the mean disk thickness $\Sigma$ are sensitive to the experimental conditions in the selected core distance range. Plots have been made for three conditions (Section 3.3.1): no inaccuracy on the core location and on the arrival time measurement $(\Delta r=0$, $\Delta t=0) ; 6 \mathrm{~m}$ accuracy of the core location and no inaccuracy on the arrival time measurement $(\Delta r=6 \mathrm{~m}$, $\Delta t=0) ; 6 \mathrm{~m}$ accuracy of core position and $2 \mathrm{~ns}$ accuracy of the arrival time measurement $(\Delta r=6 \mathrm{~m}, \Delta t=$ $2 \mathrm{~ns}$ ). It can be seen that experimental conditions do not affect the mean disk thickness $\Sigma$, while the mean arrival time $T$ is very sensitive to the accuracy on arrival time measurement. This is due to the processing of experimental data, in which the zero time in each event has been associated with the arrival time of the first particle hitting the detector. In this way fluctuations of arrival time measurements of the first particle
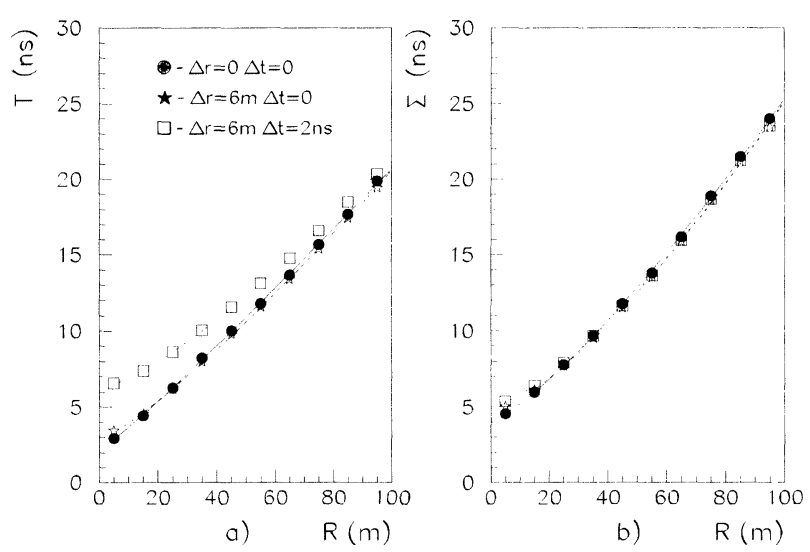

Fig. 7. (a) Mean arrival time $T$ and (b) mean disk thickness $\Sigma$ derived by averaging individual $\left\langle\tau_{i}^{k}\right\rangle$ and $\sigma^{k}$ for simulated showers in different experimental conditions as a function of the EAS core distance $R$. The lines drown through the points are the best fit obtained using function (3).

are transferred to the delay measurement of all other particles with respect to the first one, and the probability to add a significant delay to particles increases with the particle density.

\subsection{Comparison with integrated inclusive distributions}

Integrated inclusive distributions reported in [6] have been obtained by summing up delays of all particles in all events. Because selected events were common for integrated and individual distributions, results can be immediately compared. In Figs. 8a,b the mean profile $T$ and thickness $\Sigma$ derived by averaging individual $\left\langle\tau_{i}^{k}\right\rangle$ and $\sigma^{k}$ are compared with mean profile $\langle\tau\rangle$ and thickness $\sigma$ obtained from inclusive distributions. It is seen that, while mean profiles obviously coincide, the front thickness obtained by averaging individual $\sigma^{k}$ as $\Sigma=\sum_{k=1}^{n} \sigma^{k} / N$ is substantially less than that from inclusive distributions. This means that the long train of delayed particles observed in inclusive delay distributions are due to events in which delayed particles appear as a compact group with relatively small individual $\sigma^{k}$.

In order to check this conclusion, a comparison has been made between individual and integrated distributions obtained from simulations. The result is shown in Figs. 8c,d in which the mean profile $T$ and mean disk thickness $\Sigma$ for integrated distribution obtained with 

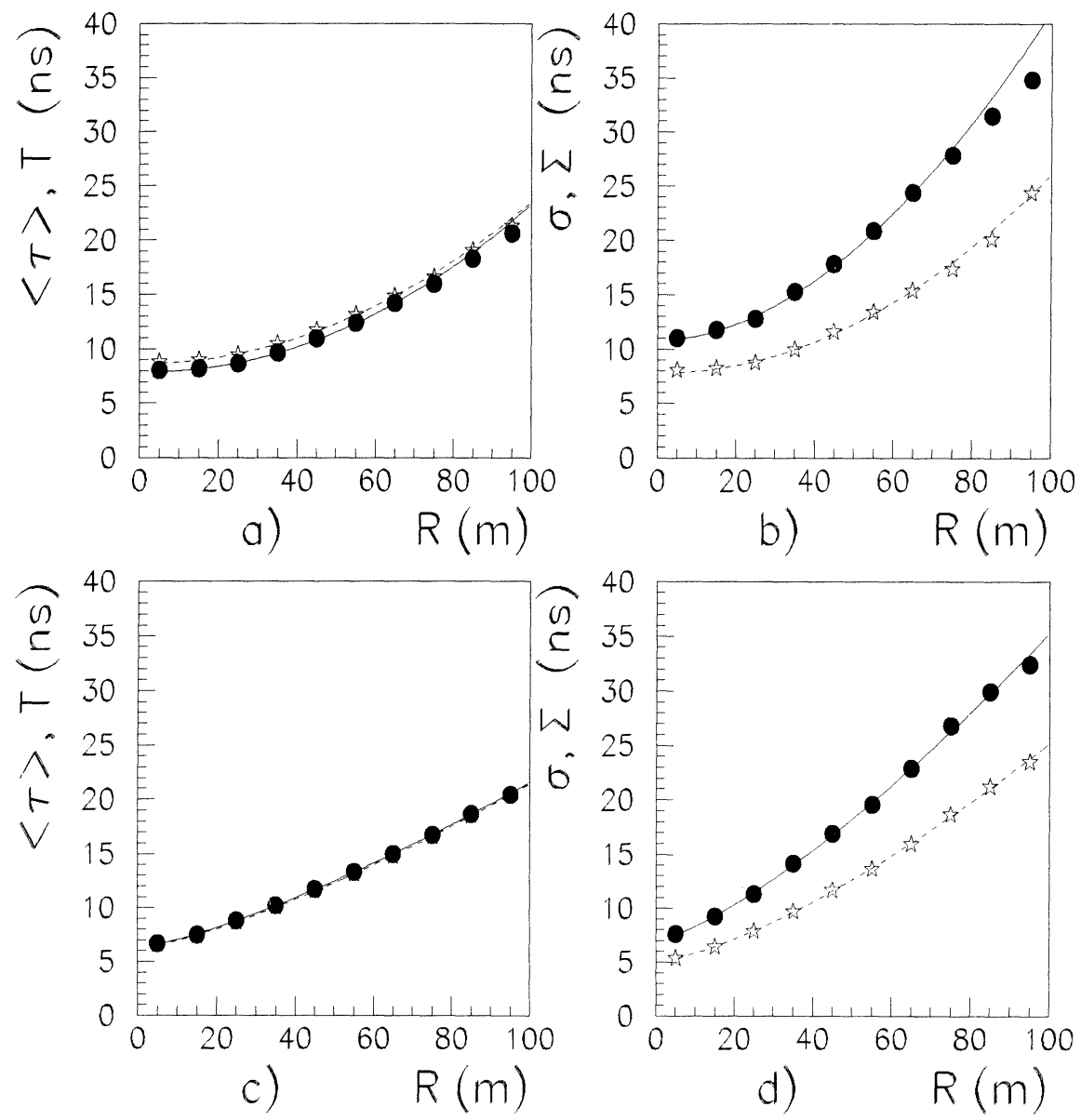

Fig. 8. Mean arrival time $(\langle\tau\rangle, T)$ and mean shower disk thickness $(\sigma, \Sigma)$ as the function of EAS core distance $R$, derived from inclusive $(\bullet)$ and individual $(\star)$ distributions for experimental data (upper plots) and simulated showers (lower plots). Lines drawn through these points are the best fit obtained using expression (3).

simulation are compared with the same quantities for simulated individual events. The difference between simulated values is identical to the difference between experimental ones. This means that the difference in the mean disk thickness between integrated and individual distributions has a common origin: it is simply due to large fluctuations in the shower longitudinal development. Such fluctuations result in the correlated delay for the bulk of the particles, which appear as a relatively compact group delayed by different time intervals from the first arriving particle. That in turn results in the different weight of delayed particles in individual distributions (few particles) and integrated inclusive distribution (a huge amount of particles).

\subsection{Comparison with previous measurements}

Present results can be compared with previous results obtained at larger core distances, where the low particle density makes possible the measurement of individual particle arrival times with respect to the light front even for a conventional array. In particular results obtained in Moscow University [25], Volcano Ranch $[9,10]$ and their extrapolation to the core region attempted by Linsley [7] on the basis of [8] can be compared with the present results. Fig. 9a shows the mean disk thickness at large core distances derived from present simulations, MSU and Volcano Ranch data. The superimposed curve is the fit of only simulated data according to (3) with the best fit param- 

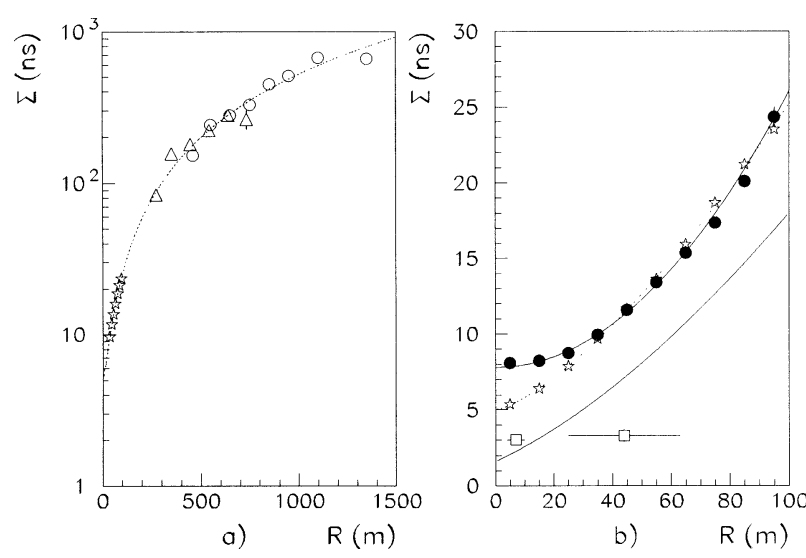

Fig. 9. Mean disk thickness: (a) at large core distances, taken from our simulations $(\star)$, MSU $(\triangle)$ and Volcano Ranch experimental data $(\circ)$. The line drawn through the points is the function (3) with the best fit parameters taken from the lowest line of Table 1; (b) at smaller core distances, our experimental data $(\bullet)$, simulation results $(\star)$ and Kiel data $(\square)$ with the superimposed Linsley's expression as the lower full line.

eters taken from the lowest line of Table 1 . It can be seen that our simulations are in good agreement with MSU and Volcano Ranch data. The front thickness can be parameterized by expression (3) with parameters taken from Table 1. For smaller core distances the present experimental data and simulation results are presented in Fig. 9b. The observed discrepancy at small core distances is probably due to the presence, near the core, of low energy hadrons not abundant at large core distances. In the same plot the experimental data from Kiel array [8] are also presented with the superimposed Linsley's expression [7] as the lower full line. It is seen that this parametrization matches neither the present experimental data nor simulation results in $0-100 \mathrm{~m}$ core distance range.

On the other hand, the ability of (3) to describe the EAS disk thickness as the function of core distance has been confirmed by recent measurements of the muon arrival times performed in KASCADE [28]. This means that formula (3) is a general formula describing the EAS front thickness at any core distance: it fails only close to the shower core $(R<40 \mathrm{~m})$ if hadrons are detected, because low energy hadrons make the disk flattter. CORSIKA 4.50 Monte Carlo cannot reproduce this effect because it cannot simulate reliably hadrons with an energy less than $50 \mathrm{MeV}$. There are measurements of the temporal EAS disk structure in Akeno [26,27]. However, Japanese physi- cists used as the disk width parameter the values of $T_{10-50}$ or $T_{10-80}$ - the time interval between $10 \%$ and $50 \%$ or $80 \%$ of the full integrated signal. The late starts and long tails of the arrival time distribution are biased by the use of these parameters and fluctuations are substantially reduced. That is why the standard deviation of their $T_{10-80}$ distribution is less than our $\Sigma: 4.0 \mathrm{~ns}$ versus $16.6 \mathrm{~ns}$ at $R=70 \mathrm{~m}$ [26]. Nevertheless, even these reduced fluctuations are rather large. The spread of $T_{10-50}$ values presented in [27] for core distances $\mathrm{R}>300 \mathrm{~m}$ complements our conclusion about large fluctuations of the EAS disk width at distances $R<$ $100 \mathrm{~m}$, which makes the idea to determine the core distance $R$ by measuring the individual time width $\sigma^{k}$ unrealistic in the whole range of EAS core distances.

\section{Discussion}

Arrival time distributions of individual particles in individual EAS show that the shower front appears as a narrow bunch of particles followed by a long train of particles delayed up to $100 \mathrm{~ns}$ with respect to the first arrival (Fig. 3). The bulk of this distribution is well described by a $\Gamma$-p.d.f. in a core distance range of 0-100 m [6]. Close to the shower core (0-30 m) contribution of low energy hadrons, below $50 \mathrm{MeV}$, makes the bunch larger and the average $\left\langle\tau_{i}^{k}\right\rangle$ and $\sigma^{k}$ as the function of $R$ flatter (Fig. 6). Delayed particles are mostly concentrated in a minor fraction of events which are present both in experimental and in simulated data (Fig. 8). Simulations reproduce experimental results quite well, except at core distances less than $30 \mathrm{~m}$ because the CORSIKA 4.50 Monte Carlo code is unable to follow low energy hadrons abundant mostly near the shower core.

Distributions are not significantly dependent on the energy and nature of the primary particle, neither on the shower axis inclination in the selected core distance and particle density range. On the other hand, distributions are very sensitive to the accuracy of the arrival time measurement and electronics time resolution (Fig. 7).

The average arrival time $\left\langle\tau_{i}^{k}\right\rangle$ of particles in the shower front was found to be weakly correlated with the front thickness $\sigma^{k}$ (Fig. 5) at any core distance.

Very impressive is the presence in experimental data of events with relatively high $\left\langle\tau_{i}^{k}\right\rangle$ and small $\sigma^{k}$ oc- 
cupying the same area in Fig. 5 as delayed showers reported in [24]. Simulations do not reproduce this kind of events, reducing to $10^{-3}$ the probability of delayed showers being due to sporadic fluctuations of the arrival time of particles in the shower front. We cannot exclude that the lack of such events in simulations is due to some technical reasons, the same or similar to the absence in CORSIKA of hadrons and muons with energies below $50 \mathrm{MeV}$. However, in our mind the delayed showers are most likely connected with the large fluctuations in the longitudinal shower development. This hypothesis has to be checked with the help of larger and more complete simulations.

Comparison of present measurements and simulations with MSU [25] and Volcano Ranch [9] data allows the parameterization of shower front thickness in a core distance range up to $1500 \mathrm{~m}$. The expression (3) with parameters of Table 1 fits well both the present data, MSU and Volcano Ranch data with a small deviation in the $0-30 \mathrm{~m}$ interval in which the shower thickness is dominated by low energy hadrons.

\section{Conclusion}

The use of highly segmented RPC embedded in the GREX array permitted a detailed study of the individual EAS front time structure in the core distance range of $0-100 \mathrm{~m}$. Characteristics of mean arrival time and front thickness distributions have been analysed and compared with CORSIKA 4.50 Monte Carlo simulations.

The dependence of the mean arrival time and average front thickness in individual showers on the core distance has been compared with those derived from integrated inclusive distributions. It has been found that the width of the individual distribution is systematically less than that of the inclusive distribution. It means that the bulk of particles in individual showers arrives as a relatively compact group delayed by different time intervals from the first arriving particle. Such fluctuations of the arrival time for most of the shower particles may be the consequence of large fluctuations in the shower longitudinal development.

The study of the correlation between the mean individual arrival time of particles and the front thickness revealed the presence of events with a large mean arrival time and a relatively small thickness not repro- duced by simulations.

Comparison with simulations revealed also a significant contribution of low energy hadrons to the width of the shower front near the core.

The performance of RPC in the G/C_P experiment demonstrated that the use of such detector in EAS physics can be very fruitful and can give precise information on shower front characteristics, thus allowing a further and detailed study of extensive air shower phenomena.

\section{Acknowledgements}

Authors are grateful to the Leeds group of Prof. A.A. Watson for the array disposition and maintenance, in particular J. Beaman, J. Lloyd-Evans, P. Ogden and M. Patel. The same gratitude is due to the Palermo group of Prof. L. Scarsi for the PLASTEX and RPC operations.

A particular thanks are to Dr. J. Knapp and Dr. D. Heck for their indispensable help in installing and running CORSIKA 4.50 Monte Carlo in Naples.

\section{References}

[1] D.E. Alexandreas et al., 23th Int. Cosm. Ray Conf., Calgary, Vol. 1 (1993) p. 223.

[2] B.S. Acharya et al., 23th Int. Cosm. Ray Conf., Calgary, Vol. 1 (1993) p. 212.

[3] J. Prahl et al., 23th Int. Cosm. Ray Conf., Calgary, Vol. 4 (1993) p. 734.

[4] A.V. Plyasheshnikov, 22th Int. Cosm. Ray Conf., Dublin, Vol. 1 (1991) p. 488.

[5] P. Bassi, G. Clark, B. Rossi, Phys. Rev. 92 (1953) 441.

[6] G. Agnetta et al., Astropart. Phys. 6 (1997) 301.

[7] J. Linsley, J. Phys. G 12 (1986) 51.

[8] C.P. Woidneck, E. Böhm, J. Phys. A 8 (1975) 997.

[9] J. Linsley, L. Scarsi, B. Rossi, Phys. Rev. Lett. 6 (1961) 485.

[10] J. Linsley, L. Scarsi, Phys. Rev. 128 (1962) 2384.

[11] H. Sakuyama et al., Nuovo Cimento C 6 (1983) 371.

[12] H. Sakuyama et al., Nuovo Cimento A 78 (1983) 147.

[13] M. Yoshida et al., J. Phys. Soc. Jpn 53 (1984) 1983.

[14] G.B. Khristiansen et al., 21th Int. Cosm. Ray Conf., Adelaide, Vol. 9 (1990) p. 150.

[15] R. Santonico, R. Cardarelli, Nucl. Instr. \& Meth. 187 (1981) 377.

[16] R. Santonico, R. Cardarelli, Nucl. Instr. \& Meth. A 236 (1988) 20.

[17] G. Brooke et al., 19th ICRC, La Jolla, Vol. 3 (1985) 426.

[18] G. Agnetta et al., Nuovo Cimento C 13 (1990) 391. 
[19] J.N. Capdevielle et al., Kernforsch. Karlsruhe, KFK 4998 (1992).

[20] M. Ambrosio et al., Nucl. Instr. \& Meth. A 334 (1994) 350.

[21] G. Agnetta et al., Nucl. Instr. \& Meth. A 381 (1996) 64.

[22] G. Agnetta et al., Nucl. Instr. \& Meth. A 315 (1992) 415.

[23] G. Agnetta et al., Nucl. Instr. \& Meth. A 337 (1994) 521.

[24] M. Ambrosio et al., Nucl. Phys. B (Proc. Suppl.) 52 (1997)
234.

[25] V.B. Atrashkevich et al., 20th Int. Cosm. Ray Conf., Moscow, Vol. 6 (1987) p. 63.

[26] N. Inoue et al., J. Phys. G 15 (1989) 1899.

[27] K. Honda et al., 23d Int. Cosm. Ray Conf., Calgary, Vol. 4 (1993) 311.

[28] U. Raidt, Internal Report FZKA 5917, Forschungszentrum Karlsruhe (August 1997). 